\title{
Intralipid treatment: Is it only the tip of an iceberg? A new suggestion: Bone cement implantation syndrome (BCIS)
}

\author{
Joseph Eldor ${ }^{1 *}$, Vladimir Kotlovker ${ }^{1}$ \\ Correspondence: csen international@csen.com \\ 'Theoretical Medicine Institute, Jerusalem 91273, Israel.
}

\begin{abstract}
On 1998 it was first showed that intravenous intralipid could prevent or improve resuscitation from cardiovascular collapse by severe bupivacaine overdose in rats.

Since then published examples now include toxicities related to verapamil, diltiazem, amlodipine, quetiapine and sertraline, haldoperidol, lamotrigine, olanzapine, propranolol, atenolol, nevibolol, doxepin, dosulepin, imipramine, amitriptyline, glyosphate herbicide, flecainide, venlafaxine, moxidectin, and others.

Intralipid treatment is a new treatment for Bone cement implantation syndrome (BCIS) which was never suggested before. Animal studies should be done in order to evaluate this new treatment modality.
\end{abstract}

Keywords: Intralipid, bupivacaine toxicity, bone cement implantation syndrome (BCIS)

Pretreatment or resuscitation with a lipid infusion Intralipid ${ }^{\circledR} 10 \%$ (10\% i.v fat emulsion) (a 10\% intravenous fat emulsion) is a sterile, non-pyrogenic fat emulsion prepared for intravenous administration as a source of calories and essential fatty acids. it is made up of $10 \%$ soybean oil, $1.2 \%$ egg yolk phospholipids, $2.25 \%$ glycerin, and water for injection. in addition, sodium hydroxide has been added to adjust the ph so that the final product ph is 8 . ph range is 6 to 8.9 .

The soybean oil is a refined natural product consisting of a mixture of neutral triglycerides of predominantly unsaturated fatty acids. the major component fatty acids are linoleic $(44-62 \%)$, oleic (19-30\%), palmitic (7-14\%), linolenic (4-11\%) and stearic (1.4-5.5\%).

Purified egg phosphatides are a mixture of naturally occurring phospholipids which are isolated from the egg yolk.

Intralipid ${ }^{\oplus} 10 \%$ (10\% i.v fat emulsion) (a 10\% intravenous fat emulsion) has an osmolality of approximately 300 mosmol/kg water (which represents 260 mosmol/liter of emulsion) and contains emulsified fat particles of approximately 0.5 micron size.

The total caloric value, including fat, phospholipid and glycerin, is $1.1 \mathrm{kcal}$ per $\mathrm{ml}$ of intralipid $10 \%$ (10\% i.v fat emulsion). the phospholipids present contribute 47 milligrams or approximately $1.5 \mathrm{mmol}$ of phosphorus per $100 \mathrm{ml}$, of the emulsion.

Weinberg et al., [1] first showed in 1998 that an infusion of a soybean oil emulsion normally used as a total parenteral nutrition solution could prevent (by pretreatment) or improve resuscitation from cardiovascular collapse caused by severe bupivacaine overdose in the intact, anesthetized rat. Subsequent studies from the same laboratory confirmed these findings in isolated rat heart [2] and anesthetized dog [3]. Under the latter experimental model, return of spontaneous circulation after a bupivacaine challenge occurred in all animals receiving lipid, but in none of the saline controls [3]. This study was accompanied by an editorial asking whether lipid might be the long-sought "silver bullet" for local anesthetic systemic toxicity (LAST). Since then, the effectiveness of lipid emulsion infusion in reversing LAST has been confirmed in other laboratories and by systematic analysis [4] in the clinical setting as well.

Lipid infusion is useful in reversing cardiac toxicity of local anesthetics, and recent reports indicate it may be useful in resuscitation from toxicity induced by a variety of other drugs. While the mechanism behind the utility of lipid rescue remains to be fully elucidated, the predominant effect appears to be creation of a "lipid sink".

French $\mathrm{D}$ et al., [5] tried to determine whether the extraction of drugs by lipid, and hence the clinical efficacy of lipid rescue in toxicological emergencies can be predicted by specific drug properties.

Each drug investigated was added individually to human drug-free serum. Intralipid ${ }^{\circledR}$ was added to this drug-containing serum, shaken and then incubated at $37^{\circ} \mathrm{C}$. The lipid was removed by ultracentrifugation and the concentration of drug remaining in the serum was measured by high-pressure liquid chromatography. 
In this in vitro model, the ability of lipid emulsion to bind a drug was largely dependent upon the drug's lipid partition constant. Additionally, using a multiple linear regression model, the prediction of binding could be improved by combining the lipid partition constant with the volume of distribution together accounting for approximately $88 \%$ of the variation in the decrease in serum drug concentration with the administration of lipid emulsion.

The lipid partition constant and volume of distribution can likely be used to predict the efficacy of lipid infusion in reversing the cardiac toxicity induced by anesthetics or other medications.

Local anaesthetics may induce cardiac arrest, usually because of rapid absorption from the site of injection or because of an intended intravascular injection. Early central nervous system symptoms usually precede seizures. Cardiac arrhythmias follow the CNS signs. These arrhythmias often resolve with the i.v. bolus injection of 100 to $150 \mathrm{~mL}$ of a lipid emulsion (20\% Intralipid $\left.\left({ }^{(}\right)\right)$. Although long acting local anaesthetics (bupivacaine, ropivacaine, levobupivacaine) are predominantly involved in this cardiac toxicity, lidocaine may also induce cardiac arrhythmias and clinician must be aware of this risk. In case of cardiac arrest, resuscitation manoeuvres are of major importance. They need to be performed immediately and the efficacy of the lipid rescue requires a correct coronary flow to be efficacious. Finally, prevention is the key of a safe injection. It is important to control the dose, to inject slowly, without any excessive pressure and to verify that no blood reflux occurs [6].

\section{Reversing other drugs toxicity}

These publications, along with other animal studies, opened the door to more widespread use of lipid emulsion for emergency treatment of toxicities caused by a range of lipophilic drugs. Notably, published examples now include toxicities related to verapamil, diltiazem, amlodipine, quetiapine and sertraline, haldoperidol, lamotrigine, olanzapine, propranolol, atenolol, nevibolol, doxepin, dosulepin, imipramine, amitriptyline, glyosphate herbicide, flecainide, venlafaxine, moxidectin, and others.

Tricyclic antidepressant (TCA) toxicity results predominantly from myocardial sodium-channel blockade. Subsequent ventricular dysrhythmias, myocardial depression, and hypotension cause cardiovascular collapse. Animal studies have demonstrated the effectiveness of intravenous lipid-emulsion in treating TCA cardiotoxicity.

Blaber MS et al., [7] report a case of dothiepin (tricyclic antidepressant) overdose causing refractory cardiovascular collapse, which seemed to be successfully reversed with lipid-emulsion therapy (Intralipid ${ }^{\oplus}$; Fresenius, Cheshire, UK).

Lipid emulsions are a potentially novel therapy for reversing cardiotoxicity seen in TCA overdose. Research is required into the role of lipid emulsion in the management of poisoning by oral lipophilic agents.

\section{The lipid sink effect}

Papadopoulou A et al., [8] hypothesized that by substituting a dye surrogate in place of local anesthetic, they could visually demonstrate dye sequestration by lipid emulsion that would be dependent on both dye lipophilicity and the amount of lipid emulsion used.

They selected 2 lipophilic dyes, acid blue 25 and Victoria blue, with log $P$ values comparable to lidocaine and bupivacaine, respectively. Each dye solution was mixed with combinations of lipid emulsion and water to emulate "lipid rescue" treatment at dye concentrations equivalent to fatal, cardiotoxic, and neurotoxic local anesthetic plasma concentrations. The lipid emulsion volumes added to each dye solution emulated equivalent intravenous doses of 100,500 , and $900 \mathrm{~mL}$ of $20 \%$ Intralipid in a $75-\mathrm{kg}$ adult. After mixing, the samples were separated into a lipid-rich supernatant and a lipid-poor subnatant by heparin flocculation. The subnatants were isolated, and their colors compared against a graduated dye concentration scale.

Lipid emulsion addition resulted in significant dye acquisition by the lipid compartment accompanied by a reduction in the color intensity of the aqueous phase that could be readily observed. The greatest amount of sequestration occurred with the dye possessing the higher $\log P$ value and the greatest amount of lipid emulsion.

This study provides a visual demonstration of the lipid sink effect. It supports the theory that lipid emulsion may reduce the amount of free drug present in plasma from concentrations associated with an invariably fatal outcome to those that are potentially survivable.

Local anesthetic (LA) intoxication with cardiovascular arrest is a potential fatal complication of regional anesthesia. Lipid resuscitation has been recommended for the treatment of LA-induced cardiac arrest. Aim of the study [9] was to compare four different rescue regimens using epinephrine and/or lipid emulsion and vasopressin to treat cardiac arrest caused by bupivacaine intoxication.

Twenty-eight piglets were randomized into four groups $(4 \times 7)$, anesthetized with sevoflurane, intubated, and ventilated. Bupivacaine was infused with a syringe driver via central venous catheter at a rate of $1 \mathrm{mg} \cdot \mathrm{kg}(-1) \cdot \mathrm{min}(-1)$ until circulatory arrest. Bupivacaine infusion and sevoflurane were then stopped, chest compression was started, and the pigs were ventilated with $100 \%$ oxygen. After 1 min, epinephrine $10 \mu \mathrm{g} \cdot \mathrm{kg}(-1)$ (group 1), Intralipid( $\left.{ }^{\oplus}\right) 20 \% 4 \mathrm{ml} \cdot \mathrm{kg}(-1)$ (group 2), epinephrine $10 \mu \mathrm{g} \cdot \mathrm{kg}(-1)+\operatorname{Intralipid}\left({ }^{\oplus}\right) 4 \mathrm{ml} \cdot \mathrm{kg}(-1)$ (group 3) or 2 IU vasopressin + Intralipid( $\left.{ }^{(}\right) 4 \mathrm{ml} \cdot \mathrm{kg}(-1)$ (group 4) were administered. Secondary epinephrine doses were given after $5 \mathrm{~min}$ if required.

Survival was $71 \%, 29 \%, 86 \%$, and $57 \%$ in groups $1,2,3$, and 4. Return of spontaneous circulation was regained only by initial administration of epinephrine alone or in combination with Intralipid( $\left({ }^{\oplus}\right)$. Piglets receiving the combination therapy survived without further epinephrine support. In contrast, in groups 2 and 4, return of spontaneous circulation was 
only achieved after secondary epinephrine rescue.

In cardiac arrest caused by bupivacaine intoxication, firstline rescue with epinephrine and epinephrine + Intralipid $\left({ }^{\circledR}\right)$ was more effective with regard to survival than Intralipid $\left.{ }^{(}\right)$ alone and vasopressin + Intralipid $\left(^{\circledR}\right)$ in this pig model (9).

Local anesthetic (LA) intoxication with severe hemodynamic compromise is a potential catastrophic event. Lipid resuscitation has been recommended for the treatment of LA-induced cardiac arrest. However, there are no data about effectiveness of Intralipid for the treatment of severe cardiovascular compromise prior to cardiac arrest. Aim of this study was to compare effectiveness of epinephrine and Intralipid for the treatment of severe hemodynamic. Piglets were compromise owing to bupivacaine intoxication, anesthetized with sevoflurane, intubated, and ventilated. Bupivacaine was infused with a syringe driver via a central venous catheter at a rate of $1 \mathrm{mg} \cdot \mathrm{kg}(-1) \cdot \min (-1)$ until invasively measured mean arterial pressure (MAP) dropped to $50 \%$ of the initial value. Bupivacaine infusion was then stopped, and epinephrine $3 \mu \mathrm{g} \cdot \mathrm{kg}(-1)$ (group 1), Intralipid $\left.{ }^{(}\right)$ $20 \% 2 \mathrm{ml} \cdot \mathrm{kg}(-1)$ (group 2), or Intralipid 20\% $4 \mathrm{ml} \cdot \mathrm{kg}(-1)$ (group 3) was immediately administered. Twenty-one piglets $(3 \times 7)$, were recorded. All animals in group $1(100 \%)$ but only four of seven (57\%) piglets in group 2 and group 3 , respectively, survived. Normalization of hemodynamic parameters (HR, MAP) and ET(CO2) was fastest in group 1 with all piglets achieving $\mathrm{HR}$ and MAP values. Hemodynamic compromise owing to bupivacaine intoxication in piglets, first-line rescue with epinephrine was more effective than Intralipid with regard to survival as well as normalization of hemodynamic parameters and ET(CO2) [10].

Intravenous lipid emulsion (ILE) has been proposed as a rescue therapy for severe local anesthetic drugs toxicity, but experience is limited with other lipophilic drugs. An 18-year-old healthy woman was admitted $8 \mathrm{~h}$ after the voluntary ingestion of sustained-release diltiazem (3600 $\mathrm{mg})$, with severe hypotension refractory to fluid therapy, calcium salts, and high-dose norepinephrine $(6.66 \mu \mathrm{g} / \mathrm{kg} /$ min). Hyperinsulinemic euglycemia therapy was initiated and shortly after was followed by a protocol of ILE (intralipid 20\%, $1.5 \mathrm{ml} / \mathrm{kg}$ as bolus, followed by $0.25 \mathrm{ml} / \mathrm{kg}$ over $1 \mathrm{~h}$ ). The main finding attributed to ILE was an apparent rapid decrease in insulin resistance, despite a prolonged serum diltiazem elimination half-life. Diltiazem is a lipophilic cardiotoxic drug, which could be sequestered in an expanded plasma lipid phase. The mechanism of action of ILE is not known, including its role in insulin resistance and myocardial metabolism in calcium-channel blocker poisoning [11].

\section{Guidelines for the management of local anaesthetic toxicity}

There is increasing evidence for the use of Intralipid in the management of acute local anaesthetic toxicity. This is supported by the recent Association of Anaesthetists of Great Britain and Ireland (AAGBI) guidelines for the management of local anaesthetic toxicity. Acute hospitals in England and Wales were surveyed to determine the proportion that currently stocked Intralipid, the locations of stocks within the hospital, guidelines related to its use and previous use in the last 12 months. The majority of hospitals surveyed stocked Intralipid in multiple locations, although not in all areas using high volumes of local anaesthetics. Guidelines were typically in place, although these were often local rather than those from the AAGBI. Use in the last 12 months was uncommon, but typically information was not available on indications for its use. More systematic data collection is required on the safety and efficacy of Intralipid in the management of acute drug toxicity [12].

Intralipid therapy has been used successfully as "rescue therapy" in several cases of overdose. West PL et al., [13] present a case of iatrogenic lipid emulsion overdose because of a dosing error: "A 71-year-old female overdosed on 27 tablets of $5 \mathrm{mg}$ amlodipine. Although initially stable in the Emergency Department, she became hypotensive, oliguric, and respiratory failure developed despite medical therapy. The primary treating team felt that meaningful recovery was unlikely to occur without rapid improvement in clinical status, and $12.5 \mathrm{~h}$ after presentation, intralipid rescue therapy was initiated. A protocol for intralipid specifying a maximum infusion of $400 \mathrm{~mL}$ of $20 \%$ lipid emulsion was faxed, but the infusion was continued until $2 L$ of lipid emulsion was infused. There were no detectable adverse hemodynamic effects of the intralipid infusion. After this time, laboratory values were difficult to obtain. Three hours after the infusion, a metabolic panel was obtained from ultracentrifuged blood showing hyponatremia. A white blood cell (WBC) was obtained from a complete blood count (CBC) performed $22 \mathrm{~h}$ after the infusion, hemoglobin and hematocrit could not be obtained from this blood. A platelet count was obtained by smear estimate. Hematocrits were obtained from centrifuged blood and appeared elevated. No oxygenation could be obtained on blood gas. The patient's family chose to withdraw care on hospital day 2 and no further laboratory draws were obtained. Amlodipine was 1,500 ng/mL (ref. 3-11ng/mL)."

\section{Lipid emulsion overdose}

Lipid emulsion overdose caused no detectable acute adverse hemodynamic effects. The following laboratory values were unobtainable immediately after infusion: white blood cell count, hemoglobin, hematocrit, platelet count, and a metabolic panel of serum electrolytes. Ultracentrifugation of blood allowed for detection of a metabolic panel $3 \mathrm{~h}$ after the infusion. Centrifuged hematocrits appeared to be higher than expected.

Lipid infusion reverses systemic local anesthetic toxicity. The acceptable upper limit for lipid administration is unknown and has direct bearing on clinical management. Hiller DB et al., [14] hypothesize that high volumes of lipid could have undesirable effects and sought to identify the 
dose required to kill $50 \%$ of the animals ( $L D(50))$ of large volume lipid administration.

Intravenous lines and electrocardiogram electrodes were placed in anesthetized, male Sprague-Dawley rats. Twenty percent lipid emulsion $(20,40,60$, or $80 \mathrm{~mL} / \mathrm{kg}$ ) or saline $(60$ or $80 \mathrm{~mL} / \mathrm{kg})$, were administered over 30 mins; lipid dosing was assigned by the Dixon "up-and-down" method. Rats were recovered and observed for $48 \mathrm{hrs}$ then euthanized for histologic analysis of major organs. Three additional rats were administered $60 \mathrm{~mL} / \mathrm{kg}$ lipid emulsion and euthanized at 1, 4, and 24 hrs to identify progression of organ damage.

The maximum likelihood estimate for $L D(50)$ was 67.72 $(\mathrm{SE}, 10.69) \mathrm{mL} / \mathrm{kg}$. Triglycerides were elevated immediately after infusion but returned to baseline by $48 \mathrm{hrs}$ when laboratory abnormalities included elevated amylase, aspartate aminotransferase, and serum urea nitrogen for all lipid doses. Histologic diagnosis of myocardium, brain, pancreas, and kidneys was normal at all doses. Microscopic abnormalities in lung and liver were observed at 60 and $80 \mathrm{~mL} / \mathrm{kg}$; histopathology in the lung and liver was worse at $1 \mathrm{hr}$ than at 4 and $24 \mathrm{hrs}$.

The $L D(50)$ of rapid, high volume lipid infusion is an order of magnitude greater than doses typically used for lipid rescue in humans and supports the safety of lipid infusion at currently recommended doses for toxin-induced cardiac arrest. Lung and liver histopathology was observed at the highest infused volumes.

Cave $G$ and Harvey M [15] evaluate the efficacy of lipid emulsion as antidotal therapy outside the accepted setting of local anesthetic toxicity.

\section{Intralipid rescue: 1966-2009}

Literature was accessed through PubMed, OVID (1966-February 2009), and EMBASE (1947-February 2009) using the search terms "intravenous" AND ["fat emulsion" OR "lipid emulsion" OR "Intralipid"] AND ["toxicity" OR "resuscitation" OR "rescue" OR "arrest" OR "antidote"]. Additional author and conference publication searches were undertaken. Publications describing the use of lipid emulsion as antidotal treatment in animals or humans were included.

Fourteen animal studies, one human study, and four case reports were identified. In animal models, intravenous lipid emulsion (ILE) has resulted in amelioration of toxicity associated with cyclic antidepressants, verapamil, propranolol, and thiopentone. Administration in human cases has resulted in successful resuscitation from combined bupropion/lamotrigine-induced cardiac arrest, reversal of sertraline/quetiapine-induced coma, and amelioration of verapamil- and beta blocker-induced shock.

Management of overdose with highly lipophilic cardiotoxic medications should proceed in accord with established antidotal guidelines and early poisons center consultation. Data from animal experiments and human cases are limited, but suggestive that ILE may be helpful in potentially lethal cardiotoxicity or developed cardiac arrest attributable to such agents. Use of lipid emulsion as antidote remains a nascent field warranting further preclinical study and systematic reporting of human cases of use.

Previous investigators have demonstrated amelioration of lipid-soluble drug toxidromes with infusion of lipid emulsions. Clomipramine is a lipid-soluble tricyclic antidepressant with significant cardiovascular depressant activity in human overdose. Harvey M and Cave G [16] compare resuscitation with Intralipid versus sodium bicarbonate in a rabbit model of clomipramine toxicity.

Thirty sedated and mechanically ventilated New Zealand White rabbits were infused with clomipramine at $320 \mathrm{mg} /$ $\mathrm{kg}$ per hour. At target mean arterial pressure of $50 \%$ initial mean arterial pressure, animals were rescued with $0.9 \%$ $\mathrm{NaCl} 12 \mathrm{~mL} / \mathrm{kg}, 8.4 \%$ sodium bicarbonate $3 \mathrm{~mL} / \mathrm{kg}$, or $20 \%$ Intralipid $12 \mathrm{~mL} / \mathrm{kg}$. Pulse rate, mean arterial pressure, and QRS duration were sampled at 2.5-minute intervals to 15 minutes. In the second phase of the experiment, 8 sedated and mechanically ventilated rabbits were infused with clomipramine at $240 \mathrm{mg} / \mathrm{kg}$ per hour to a mean arterial pressure of $25 \mathrm{~mm} \mathrm{Hg}$. Animals received either $2 \mathrm{~mL} / \mathrm{kg} 8.4 \%$ sodium bicarbonate or $8 \mathrm{~mL} / \mathrm{kg} \mathrm{20 \%}$ Intralipid as rescue therapy. External cardiac compression and intravenous adrenaline were administered in the event of cardiovascular collapse.

Mean difference in mean arterial pressure between Intralipid- and saline solution-treated groups was 21.1 $\mathrm{mm} \mathrm{Hg}$ ( $95 \%$ confidence interval [Cl] 13.5 to $28.7 \mathrm{~mm} \mathrm{Hg}$ ) and $19.5 \mathrm{~mm} \mathrm{Hg}(95 \% \mathrm{Cl} 10.5$ to $28.9 \mathrm{~mm} \mathrm{Hg})$ at 5 and 15 minutes, respectively. Mean difference in mean arterial pressure between Intralipid- and bicarbonate-treated groups was $19.4 \mathrm{~mm} \mathrm{Hg}(95 \% \mathrm{Cl} 18.8$ to $27.0 \mathrm{~mm} \mathrm{Hg})$ and $11.5 \mathrm{~mm} \mathrm{Hg}(95 \% \mathrm{Cl} 2.5$ to $20.5 \mathrm{~mm} \mathrm{Hg})$ at 5 and 15 minutes. The rate of change in mean arterial pressure was greatest in the Intralipid-treated group at 3 minutes $(6.2 \mathrm{~mm} \mathrm{Hg} /$ $\min [95 \% \mathrm{Cl} 3.8$ to $8.6 \mathrm{~mm} \mathrm{Hg} / \mathrm{min}] \mathrm{In}$ the second phase of the experiment spontaneous circulation was maintained in all Intralipid-treated rabbits $(n=4)$. All animals in the bicarbonate-treated group developed pulseless electrical activity and proved refractory to resuscitation at 10 minutes $(n=4, P=.023)$.

In this rabbit model, Intralipid infusion resulted in more rapid and complete reversal of clomipramineinduced hypotension compared with sodium bicarbonate. Additionally, Intralipid infusion prevented cardiovascular collapse in a model of severe clomipramine toxicity.

\section{Bone cement implantation syndrome (BCIS)}

Bone cement implantation syndrome (BCIS) is characterised by hypoxia, hypotension and loss of consciousness occurring early after bone cementation. The haemodynamic perturbations during BCIS have not been extensively studied, particularly not in patients with femoral neck 
fracture. Kotyra $M$ et al., [17] evaluated the effects of cemented hemiarthroplasty, in these patients, on pulmonary haemodynamics, right ventricular performance, intrapulmonary shunting and physiological dead space.

Fifteen patients undergoing cemented hemiarthroplasty because of femoral neck fracture were included. Surgery was performed under total intravenous anaesthesia in the lateral position. All patients were catheterised with a radial and pulmonary artery catheter, for continuous measurements of mean arterial pressure (MAP), pulmonary arterial pressure (PAP), cardiac output, mixed venous oxygen saturation, right ventricular end-diastolic volume (RVEDV) and right ventricular ejection fraction (RVEF). Haemodynamic measurements and blood gas analyses were performed after induction of anaesthesia, during surgical stimulation before and immediately after bone cementation and prosthesis insertion, 10 and 20 min after insertion and during skin closure.

After bone cementation and prosthesis insertion, MAP $(-10 \%)$, cardiac index $(-10 \%)$ and stroke volume index $(-10 \%)$ decreased, while PAPs (10-15\%) and the pulmonary vascular resistance index (45\%) increased. RVEF decreased by 10 $20 \%$, while the RVEDV index increased by $10 \%$. Pulmonary haemodynamic and RV variables changed progressively with time, while intra-pulmonary shunting and physiological dead space increased immediately after prosthesis insertion and then returned to baseline.

Cemented hemiarthroplasty in patients with femoral neck fracture causes a pronounced pulmonary vasoconstriction and an impairment of RV function accompanied by pulmonary ventilation/perfusion abnormalities.

Acute hypotension, hypoxemia, cardiac arrhythmias, cardiac arrest, (or a combination of these), and sudden death are well-recognized complications of the cemented hip arthroplasty procedure. Collectively, these are known as the bone cement implantation syndrome (BCIS). The endogenous cannabinoids, anandamide (ANA) and 2-arachidonylglycerol (2-AG), are reported to be strong vasodilators and play a role in the hypotension associated with hemorrhagic and septic shock. In the present study [18], a potential role for the endogenous cannabinoids in influencing hemodynamic variables in $\mathrm{BCIS}$ was investigated. Thirty-five patients ( 35 hips) entered a prospective, randomized clinical trial. The patients were divided into two groups. Group 1 comprised 16 patients who had the component inserted using a conventional cementing technique, whereas group 2 consisted of 19 patients who had the femoral component inserted without cement. Blood samples were taken at six consecutive time points: before anesthesia, after reaming the femur, 2 min after insertion of stems with or without cement into the femur, and $10 \mathrm{~min}, 20$, and $30 \mathrm{~min}$ after stem insertion. In group 1 (with cement), the mean levels of ANA and 2-AG significantly increased after stem insertion. In a comparison of each group after stem insertion, mean ANA and 2-AG levels in group 1 also significantly differed from those in group 2 . By contrast, in group 2 (without cement) neither ANA nor 2-AG levels exhibited a significant increase or change at any point in time. In conclusion, it has been shown for the first time that endogenous cannabinoids are candidates for lipid mediators of BCIS [18].

Fallon KM et al., [19] described a case report of a cardiac arrest during a cemented hip arthroplasty procedure. Hemodynamic instability during methylmethacrylate use in arthroplasty surgery can be explained by fat embolization rather than the inherent toxicity of the monomer. A 78-yrold woman required a cemented hemiarthroplasty for a pathologic left subcapital fracture. The patient's past medical history included stable angina, diet-controlled type II diabetes and metastatic breast cancer. During the cementing of the canal and insertion of the femoral prosthesis, desaturation, hypotension and cardiac arrest occurred. The patient underwent a successful intraoperative resuscitation and was transferred to the intensive care unit where she subsequently developed disseminated intravascular coagulopathy. The patient died $24 \mathrm{hr}$ later and autopsy confirmed the cause of death as fat embolization. The deleterious cardiovascular effects of methylmethacrylate have been discussed in the literature. However, clinical evidence supports fat embolization during arthroplasty surgery as a greater determinant of hemodynamic compromise. Surgical precautions are paramount in minimizing the sequelae of Bone Implantation Syndrome and anesthetic treatment consists of supportive care.

Bone cement implantation syndrome is characterized by hypotension, hypoxemia, cardiac arrhythmias, cardiac arrest, or any combination of these complications. It may result from venous embolization that occurs in conjunction with intramedullary hypertension in the femur during insertion of the prosthesis in patients undergoing cemented total hip arthroplasty (THA). Intramedullary hypertension does not occur in patients undergoing noncemented THA. In this study, Ereth MH et al., [20] sought to compare embolization between patients undergoing cemented and noncemented THA and to determine whether this state resulted in cardiorespiratory deterioration. In this prospective investigation of 35 patients undergoing elective THA, they used transesophageal echocardiography and invasive hemodynamic monitoring, and in 12 of them, they monitored distribution of pulmonary ventilation and perfusion intraoperatively. Embolization was significantly greater after insertion of the prosthesis in patients undergoing cemented than in those undergoing noncemented THA. Cemented THA was also associated with decreased cardiac output and increased pulmonary artery pressure and pulmonary vascular resistance. Increases in ventilation-perfusion mismatching, however, could not be demonstrated 30 minutes after insertion of the femoral prosthesis. Intraoperative monitoring for embolism may 
help physicians assess patients in whom cardiorespiratory function deteriorates during THA [20].

Intralipid prevents and rescues fatal pulmonary arterial hypertension and right ventricular failure and enhances the inflammatory response to endotoxin Pulmonary arterial hypertension (PAH) is characterized by pulmonary vascular remodeling leading to right ventricular (RV) hypertrophy and failure. Intralipid (ILP), a source of parenteral nutrition for patients, contains $\gamma$-linolenic acid and soy-derived phytoestrogens that are protective for lungs and heart. Umar S. et al., [21] investigated the therapeutic potential of ILP in preventing and rescuing monocrotaline-induced PAH and RV dysfunction. PAH was induced in male rats with monocrotaline $(60 \mathrm{mg} / \mathrm{kg})$. Rats then received daily ILP ( $1 \mathrm{~mL}$ of $20 \%$ ILP per day IP) from day 1 to day 30 for prevention protocol or from day 21 to day 30 for rescue protocol. Other monocrotaline-injected rats were left untreated to develop severe PAH by day 21 or RV failure by approximately day 30. Saline or ILP-treated rats served as controls. Significant increase in RV pressure and decrease in RV ejection fraction in the RV failure group resulted in high mortality. Therapy with ILP resulted in $100 \%$ survival and prevented $\mathrm{PAH}$-induced RV failure by preserving $\mathrm{RV}$ pressure and RV ejection fraction and preventing RV hypertrophy and lung remodeling. In preexisting severe PAH, ILP attenuated most lung and RV abnormalities. The beneficial effects of ILP in PAH seem to result from the interplay of various factors, among which preservation and/ or stimulation of angiogenesis, suppression and/or reversal of inflammation, fibrosis and hypertrophy, in both lung and $\mathrm{RV}$, appear to be major contributors. In conclusion, ILP not only prevents the development of $\mathrm{PAH}$ and RV failure but also rescues preexisting severe PAH [21].

Novel anti-inflammatory effects of insulin have recently been described, and insulin therapy to maintain euglycemia suppresses the plasma levels of free fatty acids (FFA) and increases the survival of critically ill patients. Krogh-Madsen et al., [22] aimed to explore the effect of short-term high levels of plasma FFA on the inflammatory response to a low dose of endotoxin. Fourteen healthy male volunteers underwent the following two trials in a randomized crossover design: 1) continuous infusion of $20 \%$ Intralipid [0.7 ml.kg(-1).h(-1) (1.54 g/kg)] for $11 \mathrm{~h}$, and 2) infusion of isotonic saline for $11 \mathrm{~h}$ (control). In each trial, heparin was given to activate lipoprotein lipase, and an intravenous bolus of endotoxin ( $0.1 \mathrm{ng} / \mathrm{kg}$ ) was given after $6 \mathrm{~h}$ of Intralipid/saline infusion. Blood samples and muscle and fat biopsies were obtained before the Intralipid/saline infusion and before as well as after infusion of an endotoxin bolus. Plasma levels of FFA, triglycerides, and glycerol were markedly increased during the Intralipid infusion. Endotoxin exposure induced an increase in plasma levels of TNF-alpha, IL-6, and neutrophils and further stimulated gene expression of TNF-alpha and IL-6 in both skeletal muscle and adipose tissue. The systemic inflammatory response to endotoxin was significantly pronounced during Intralipid infusion. Short-term hyperlipidemia enhances the inflammatory response to endotoxin, and skeletal muscle and adipose tissue are capable of producing essential inflammatory mediators after endotoxin stimulation.

\section{Is Intralipid approved by the FDA for Local Anesthesia -induced cardiovascular collapse or resuscitation?}

As with any FDA-labeled medication, the individual Intralipid products carry with them a set of contraindications to use and adverse effects. However, the extent to which these apply in the setting of Local Anesthesia -induced cardiovascular collapse or resuscitation, for which Intralipid is not FDA-approved, remains to be defined. The general contraindication to Intralipid use is the presence of disorders of fat metabolism, other contraindications not published in the product's package insert include egg allergy and acute myocardial infarction. The use of Intralipid is cautioned in patients with anemia, severe liver disease, coagulopathies, pulmonary disease, and in patients at risk for fat embolism. The most common adverse effects from general Intralipid use are those related to contamination of the administration site and irritation of the veins likely due to other solutions co-infused with Intralipid.

Early or immediate adverse effects of Intralipid include allergic reactions, headache, somnolence, dizziness, diaphoresis, dyspnea, nausea/vomiting, hyperthermia, and hypercoagulability. More delayed adverse effects of Intralipid include thrombocytopenia, jaundice, overloading syndrome, increased liver function tests, leucopenia, hepatomegaly, and splenomegaly; pancreatitis has rarely been associated with Intralipid use. Those adverse effects that may be dose- or rate-related include pulmonary embolus or fat embolus, and pulmonary vasoconstriction may result from bolus administration of Intralipid. There were no adverse effects reported with Intralipid use in the four human case reports after Local Anesthesia -induced cardiovascular collapse, although further investigation is warranted [23].

\section{Conclusion}

Intralipid treatment is a new treatment for BCIS which was never suggested before. Animal studies should be done in order to evaluate this new treatment modality.

\section{Competing interests}

The authors declare that they have no competing interests.

\section{Publication history}

Received: 24-Aug-2012 Revised: 01-Oct-20112

Accepted: 16-Oct-2012 Published: 03-Nov-2012 


\section{References}

1. Weinberg GL, VadeBoncouer T, Ramaraju GA, Garcia-Amaro MF, Cwik MJ: Pretreatment or resuscitation with a lipid infusion shifts the dose-response to bupivacaine-induced asystole in rats. Anesthesiology 1998, 88:1071-1075. | Article

2. Weinberg GL, Ripper R, Murphy P, Edelman LB, Hoffman W, Strichartz $G$, Feinstein DL: Lipid infusion accelerates removal of bupivacaine and recovery from bupivacaine toxicity in the isolated rat heart. Reg Anesth Pain Med 2006, 31:296-303. I Article I PubMed

3. Weinberg G, Ripper R, Feinstein DL, Hoffman W: Lipid emulsion infusion rescues dogs from bupivacaine-induced cardiac toxicity. Reg Anesth Pain Med 2003, 28:198-202. I Article

4. Jamaty C, Bailey B, Larocque A, Notebaert E, Sanogo K, Chauny JM: Lipid emulsions in the treatment of acute poisoning: a systematic review of human and animal studies. Clin Toxicol (Phila) 2010, 48:127. I Article I PubMed

5. French D, Smollin C, Ruan W, Wong A, Drasner K, Wu AH: Partition constant and volume of distribution as predictors of clinical efficacy of lipid rescue for toxicological emergencies. Clin Toxicol (Phila) 2011, 49:801-809. | Article | PubMed

6. Mazoit JX: [Cardiac arrest and local anaesthetics.]. Presse Med 2012. | Article | PubMed

7. Blaber MS, Khan JN, Brebner JA, McColm R: "Lipid rescue" for tricyclic antidepressant cardiotoxicity. J Emerg Med 2012, 43:465467. | Article | PubMed

8. Papadopoulou A, Willers JW, Samuels TL, Uncles DR: The use of dye surrogates to illustrate local anesthetic drug sequestration by lipid emulsion: a visual demonstration of the lipid sink effect. Reg Anesth Pain Med 2012, 37:183-187. | Article I PubMed

9. Mauch J, Jurado OM, Spielmann N, Bettschart-Wolfensberger R, Weiss $M$ : Resuscitation strategies from bupivacaine-induced cardiac arrest. Paediatr Anaesth 2012, 22:124-129. I Article I PubMed

10. Mauch J, Martin Jurado O, Spielmann N, Bettschart-Wolfensberger R, Weiss $\mathrm{M}$ : Comparison of epinephrine vs lipid rescue to treat severe local anesthetic toxicity - an experimental study in piglets. Paediatr Anaesth 2011, 21:1103-1108. | Article | PubMed

11. Montiel V, Gougnard T, Hantson P: Diltiazem poisoning treated with hyperinsulinemic euglycemia therapy and intravenous lipid emulsion. Eur J Emerg Med 2011, 18:121-123. | Article | PubMed

12. Hamann P, Dargan PI, Parbat N, Ovaska H, Wood DM: Availability of and use of Intralipid (lipid rescue therapy, lipid emulsion) in England and Wales. Emerg Med J 2010, 27:590-592. | Article | PubMed

13. West PL, McKeown NJ, Hendrickson RG: latrogenic lipid emulsion overdose in a case of amlodipine poisoning. Clin Toxicol (Phila) 2010, 48:393-396. | Article | PubMed

14. Hiller DB, Di Gregorio G, Kelly K, Ripper R, Edelman L, Boumendjel $\mathrm{R}$, Drasner K, Weinberg GL: Safety of high volume lipid emulsion infusion: a first approximation of LD50 in rats. Reg Anesth Pain Med 2010, 35:140-144. | Article | PubMed

15. Cave G, Harvey M: Intravenous lipid emulsion as antidote beyond local anesthetic toxicity: a systematic review. Acad Emerg Med 2009, 16:815-824. | Article | PubMed

16. Harvey M, Cave G: Intralipid outperforms sodium bicarbonate in a rabbit model of clomipramine toxicity. Ann Emerg Med 2007, 49:178-185, 185 e171-174. | Article | PubMed

17. Kotyra M, Houltz E, Ricksten SE: Pulmonary haemodynamics and right ventricular function during cemented hemiarthroplasty for femoral neck fracture. Acta Anaesthesiol Scand 2010, 54:1210-1216. | Article | PubMed

18. Motobe T, Hashiguchi T, Uchimura T, Yamakuchi M, Taniguchi $\mathrm{N}$, Komiya S, Maruyama I: Endogenous cannabinoids are candidates for lipid mediators of bone cement implantation syndrome. Shock 2004, 21:8-12. | Article | PubMed

19. Fallon KM, Fuller JG, Morley-Forster P: Fat embolization and fatal cardiac arrest during hip arthroplasty with methylmethacrylate. Can J Anaesth 2001, 48:626-629. | Article | PubMed
20. Ereth MH, Weber JG, Abel MD, Lennon RL, Lewallen DG, Ilstrup DM, Rehder K: Cemented versus noncemented total hip arthroplasty-embolism, hemodynamics, and intrapulmonary shunting. Mayo Clin Proc 1992, 67:1066-1074. | PubMed

21. Umar S, Nadadur RD, Li J, Maltese F, Partownavid P, van der Laarse A, Eghbali M: Intralipid prevents and rescues fatal pulmonary arterial hypertension and right ventricular failure in rats. Hypertension 2011, 58:512-518. | Article | PubMed Abstract | PubMed Full Text

22. Krogh-Madsen R, Plomgaard P, Akerstrom T, Moller K, Schmitz O, Pedersen BK: Effect of short-term intralipid infusion on the immune response during low-dose endotoxemia in humans. Am J Physiol Endocrinol Metab 2008, 294:E371-379. | Article | PubMed

23. Felice K, Schumann $\mathrm{H}$ : Intravenous lipid emulsion for local anesthetic toxicity: a review of the literature. J Med Toxicol 2008, 4:184-191. | Article I PubMed

\section{Citation:}

Eldor J and Kotlovker V: Intralipid treatment: Is it only the tip of an iceberg? A new suggestion: Bone cement implantation syndrome (BCIS). journal of Anesthesiology and Clinical Science 2012, 1:12. http://dx.doi.org/10.7243/2049-9752-1-12 